\title{
Ropinirole Implants Reverse MPTP-Induced Parkinsonism in Rhesus Monkeys
}

\author{
Steven J. Siegel ${ }^{1}$, Lauren Nagy ${ }^{1}$, Torben Skarsfeldt ${ }^{2}$, Mark Pierce ${ }^{2}$, Carol O’Neill ${ }^{2}$, Robert Lin ${ }^{1}$, \\ Lori Langhamer ${ }^{3}$, Jeffery H. Kordower ${ }^{3}$ \\ ${ }^{1}$ Department of Psychiatry, University of Pennsylvania, Philadelphia, USA; ${ }^{2}$ NuPathe Inc., Conshohocken, USA; ${ }^{3}$ Rush Medical \\ Center, Chicago, USA. \\ Email: siegels@upenn.edu
}

Received May $2^{\text {nd }}, 2013$; revised May 28 ${ }^{\text {th }}, 2013$; accepted June $6^{\text {th }}, 2013$

Copyright (C) 2013 Steven J. Siegel et al. This is an open access article distributed under the Creative Commons Attribution License, which permits unrestricted use, distribution, and reproduction in any medium, provided the original work is properly cited.

\begin{abstract}
Purpose: We compared efficacy and side effects of ropinirole implants with oral ropinirole in parkinsonian monkeys. Methods: Twenty monkeys received injections of 1-methyl-4-phenyl-1,2,3,6-tetrahydropyridine-hydrochloride (MPTP) to render them parkinsonian. Monkeys were then placed into 3 groups based upon clinical rating scores (CRS). Group 1 received oral ropinirole and placebo implants. Group 2 receivedropinirole implants that released 1/9th of the animals' optimal daily oral dose and oral placebo. Group 3 received placebo implants and oral placebo. Monkeys were assessed for pharmacokinetic data, CRS, Global Dyskinesia Rating Scale, and skin irritation. Results: For the ropinirole implant group, the activity pattern was similar to that seen pre-MPTP; which extended through the weekends and was greater than control treated parkinsonian monkeys. Oral ropinirole yielded a high degree of variability for activity, with values following oral dosing being higher than the pre-MPTP periodbut levels similar to placebo treated parkinsonian animals during weekends, which were excluded from oral dosing. Implants and oral treatment achieved significant improvement in CRS between 11 - 60 days and 4 - 60 days respectively. Conclusion: Low dose ropinirole implants have the potential to provide continuous clinical improvement in bradykinesia with fewer "off periods" and lower risk for medicationinduced psychosis than oral medication.
\end{abstract}

Keywords: Ropinirole; Implant; Parkinson’s Disease; MPTP; Pharmacokinetic; Dyskinesia

\section{Introduction}

Parkinson's Disease (PD) is the second most prevalent neurodegenerative disease, affecting between $1 \%$ and $3 \%$ of adults over age 65 [1]. Clinical signs of motor parkinsonism manifest as bradykinesia, rigidity, resting tremor, and postural instability [2]. The symptoms are primarily the result of loss of putamenal dopamine insufficiency secondary to degeneration of neurons in the substanbtia nigra pars compacta. The majority of Parkinson's patients therefore use treatments that correct lost nigrostriatal dopamine function. Ropinirole is a dopamine agonist which acts on $\mathrm{D}_{2}$ postsynaptic receptors, and has been shown to be effective in treating Parkinson's disease symptoms in multiple randomized, placebo-controlled clinical studies [3-5]. Ropinirole is usually taken in the early stages of Parkinson's Disease [3]. The drug is found to have low rates of discontinuation, and is generally well tolerated [3].
Ropinirole, as with most currentpharmacological treatments for Parkinson's Disease, requires dosing multiple times per day. These dosing regimens often result in cycles of brief symptom remission interspersed with periods of abrupt symptom re-emergence as well as the emergence of serious treatment related side-effects such as dyskinesias which are believed to be the result of unstable levels of drug throughout the day [6]. In addition to the problems associated with administration of multiple doses per day, peak concentrations of clinically effective doses of dopamine agonists, including ropinirole, can lead to intermittent periods of iatrogenic psychosis and other psychiatric symptoms such as gambling, hyper-sexuality and aggression [6,7]. These issues indicate the need for long-acting treatments that avoid fluctuations in dopamine activity and motor function seen with intermittent treatments, with a reduction in the psychiatric side effects. The avoidance of fluctuations in peaktrough serum ropinirole concentrations could be obtained 
using sustained drug delivery methods. Therefore, we propose the use of ropinirole implants as a novel and improved method for the treatment of Parkinson's disease to meet these needs. The current formulation is a small, long-acting, slow-release, biodegradable implant that can deliver ropinirole consistently over a 2-month duration. This is achieved by combining ropinirole with biodegradable Poly-Lactoide-Co-Glycolide (PLGA) polymers to create sustained delivery using small rods inserted subcutaneously. Upon completion of the delivery of ropinirole, all materials degrade and are cleared by the body, avoiding the need for surgical removal $[8,9]$.

The study was conducted to accomplish the following goals:

- To compare the pharmacokinetic profile and efficacy of ropinirole implants with oral ropinirole to treat Parkinsonian symptoms in MPTP-treated Rhesus monkeys;

- To identify potential side-effects of ropinirole implants while in use in Parkinsonian Rhesus monkeys;

- To assess the potential for treatment of in Parkinson's Disease using ropinirole implants.

\section{Materials and Methods}

\subsection{Animals}

Adult male Rhesus Macaque monkeys between 6 and 12 $\mathrm{kg}$ were received at Rush University and held in quarantine for a minimum of 30 days. Baseline data were obtained for each monkey for the Clinical Rating Scale (CRS) (Table 1) and Activity Monitoring system (AMS) prior to the MPTP Induction Phase. Continuous activity levels were obtained using jackets with accelerometers that were removed once a week so that data could be downloaded (ACTITRAC, IM Systems, Inc.).

\subsection{MPTP Induction Phase}

During this phase the monkeys were made bilaterally Parkinsonian with the use of 1-methyl-4-phenyl-1,2,3,6tetrahydropyridine-hydrochloride (MPTP). Monkeys first received one dose of MPTP $0.3 \mathrm{mg} / \mathrm{kg}$ through intracarotid injection, rendering them unilaterally Parkinsonian, and then received approximately twice weekly injections of MPTP intravenously to be rendered bilaterally Parkinsonian. At the completion of the MPTP Induction Phase, 12 of 20 monkeys demonstrated stable parkinsonian symptoms with CRS scores between $7.0-20.5$ and continued with the experiment, and were tested for an optimal oral dose.

\subsection{Dose Finding Phase}

Monkeys received ropinirole at $0.5 \mathrm{mg} / \mathrm{kg}, 1 \mathrm{mg} / \mathrm{kg}$, and $2 \mathrm{mg} / \mathrm{kg}$ and CRS scores were obtained by an observer
Table 1. Monkey ID, weight, dosing, baseline CRS.

\begin{tabular}{ccccc}
\hline Oral (Group 1) & Monkey ID Weight & $\begin{array}{c}\text { Dose received } \\
\text { (mg/kg/day) }\end{array}$ & $\begin{array}{c}\text { Baseline } \\
\text { CRS }\end{array}$ \\
\hline & 7752 & 8.5 & 3 & $9 / / 10$ \\
& 7761 & 11.3 & 3 & 11 \\
& 7748 & 8.5 & 3 & $10.5 / / 12.5$ \\
& 7765 & 8.4 & 3 & 20.5 \\
Implant (Group 2) & 7757 & 7.2 & 0.1666 & 7 \\
& 7753 & 11.7 & 0.333 & $11 / / 10$ \\
& 7754 & 10 & 0.333 & $15.5 / / 13$ \\
& 7767 & 6.7 & 0.333 & $20.5 / / 19.5$ \\
Placebo (Group 3) & 7749 & 8 & 0 & $8 / / 7$ \\
& 7758 & 8 & 0 & 10 \\
& 7750 & 11.2 & 0 & $10 / / 11$ \\
& 7763 & 7.5 & 0 & 18.5 \\
\hline
\end{tabular}

Monkey identification with weight, dose received, and pre-treatment Clinical Rating Scale score. The monkeys each received a personalized dose of medicine as shown in the table, based on the optimal daily dose determined before the treatment period. The monkeys who received medication by means of the ropinirole implant received $1 / 9$ of the total optimal oral daily dose; monkeys in the placebo group received no medication at all. The baseline CRS shows the Clinical Rating Scale score of each monkey after the MPTP induction phase, and before the ropinirole treatment period began.

blinded to dose with the goal of determining the minimum dose that achieved a $50 \%$ reduction of the CRS score. A dose of $1 \mathrm{mg} / \mathrm{kg}$ was the optimal dose for 10 of the 12 monkeys. The remaining two monkeys had an optimal oral dose of $0.5 \mathrm{mg} / \mathrm{kg}$.

\subsection{Pharmacokinetic (PK) Determination}

The week prior to implantation of the ropinirole implant, acute PK for oral ropinirole was determined to ensure that behavioral testing was performed within two serum half-lives of oral dosing. Five monkeys were randomly chosen and used for a PK study in which three received $2.5 \mathrm{mg} / \mathrm{kg}$ oral ropinirole administered in food and two received $2.5 \mathrm{mg} / \mathrm{kg}$ ropinirole via oral gavage. Blood draws were taken at approximately $0.5,2,4,6$, and 10 hours post treatment for PK analysis.

\subsection{Experimentation Phases}

Monkeys were randomized into three dose groups with $n$ 4 per group (Groups 1, 2 and 3):

Group 1: Oral ropinirole three times per day (TID) using the monkey's lowest effective dose determined in the Dose Finding Phase. This group also received placebo implants to control for any effects of the procedure or presence of the implant.

Group 2: Implant that released ropinirole at a low 
dose (approximately 1/9th of the total daily oral dose). This group also received placebo oral treatments to control for any effects of daily TID oral procedures.

Group 3: Placebo implants and placebo oral treatments.

\subsection{Study Procedures}

A ropinirole or matching placebo implant was inserted for all monkeys to ensure the treatment was blinded to study scientists. Oral ropinirole was given at 8:00 am, 12:00 pm, and 4:00 pm in a food treat for a total daily dose of $3.0 \mathrm{mg} / \mathrm{kg} / \mathrm{day}$. Food treats were comprised of various types of fruit that were alternated so that monkeys could not anticipate an association between a particular food and medication effects. Monkeys in Group 1 received oral medicationin a food treat on Monday through Friday. Oral ropinirole was not given on Saturday or Sunday so as to evaluate any lasting effects of treatment on days during which the subjects did not receive the active treatment. Monkeys in Group 2 and Group 3 were given a food treat three times per day without ropinirole. PK data was collected at the time of the ropinirole or placebo implant. PK samples were taken just prior to implantation, at $30 \pm 10$ minutes, $5 \pm 1$ hour and approximately 24 hours post implantation. In addition, PK samples were taken once weekly throughout the study. Throughout the Experimentation Phase, all monkeys were also assessed weekly for CRS scores (Table 2), dyskinesia (Table 3), and skin irritation (Table 4) local to the site of implantation. Activity monitoring was collected throughout the experimentation phase and was evaluated for Wednesday-Friday (active treatment for Groups 1 and 2) and Saturday-Sunday (active treatment

Table 2. Clinical rating scale.

\begin{tabular}{cc}
\hline Characteristic & Points \\
\hline Posture & $0-3$ \\
Gait & $0-5$ \\
Bradykinesia & $0-5$ \\
Balance & $0-3$ \\
Tremor & $0-3$ for each arm \\
Gross Motor Skills & $0-4$ for each arm \\
Defense Reaction & $0-2$ \\
Freezing & $0-2$ \\
\hline
\end{tabular}

* Out of a total of 34 points, 0 corresponded to a normal scoring and 34 corresponded to extreme disability. The Clinical Rating Scale used to evaluate the degree of disability in each monkey. This scale was used by trained evaluators who observed the monkeys once per week throughout the study. Points are assigned for each characteristic of Parkinson's Disease on the scale and are tallied for a final score. A higher score corresponds to more severe Parkinsonian symptoms.
Table 3. Global non-human primate dyskinesia rating scale.

\begin{tabular}{cc}
\hline Score & Description \\
\hline 0 & $\begin{array}{r}\text { No evidence of dyskinesia. } \\
1\end{array}$ \\
2 & $\begin{array}{c}\text { Subtle movements suggestive of dyskinesia, but } \\
\text { could be normal. }\end{array}$ \\
3 & $\begin{array}{c}\text { Moderate dyskinesia; intermediate or even higher } \\
\text { amplitude, but not violent or extreme. }\end{array}$ \\
\hline
\end{tabular}

Non-human primate global dyskinesia rating scale used to evaluate presence of dyskinesias. The scale was used once per week on each monkey by trained evaluators. A score of zero indicates the absence of dyskinesia.

Table 4. Skin irritation assessment.

\begin{tabular}{cc}
\hline Score & Description \\
\hline 0 & No redness \\
1 & Minimal redness \\
2 & Moderate redness with sharply defined borders \\
3 & Intense redness without swelling \\
4 & Intense redness with swelling \\
5 & Intense redness with blistering/erosion \\
\hline
\end{tabular}

Skin Irritation Assessment used to evaluate the site of implantation. The skin around the site of placebo or ropinirole implant surgery was observed each week by trained evaluators and a score was recorded. A score of zero indicates the absence of irritation.

only in Group 2). Data from Mondays and Tuesdays were not evaluated as animals were chaired for behavioral testing and PK sampling these days. For activity evaluation, each monkey was fitted with a jacket containing an AMS. The AMS measured motion along its vertical and horizontal axes, digitizing acceleration signals and storing the average acceleration value calculated during each consecutive 30 second epoch, or time interval.

\section{Results}

\subsection{Pharmacokinetic Data}

Within 7 days, elevations of plasma ropinirole were apparent in monkeys given the ropinirole implant, with a peak concentration occurring at approximately 14 days after implantation. A comparison of the PK effects of ropinirole implants with those of oral ropinirole showed that plasma levels were approximately equivalent during study days 11 - 46, with implant levels declining for the remainder of the duration (Figure 1).

\subsection{Clinical Rating Scores}

Analysis of the CRS scores reveals a significant interaction of week by group, indicating that the groups' scores changed over the course of the study (pooled MS = 
19.325, $\mathrm{F}=1.796,(18,8), \mathrm{p}<0.05)$. The ropinirole implant group showed significant improvement from baseline scores on assessments from days 11 through 60 (CRS scores obtained on day 4 and weekly thereafter, $\mathrm{p}<$ 0.05 Tukey's post-hoc comparison all days). Monkeys receiving oral ropinirole showed significant improvement from baseline scores on all assessment days post baseline (CRS performed weekly between days $4-60$, p $<0.05$ Tukey's post-hoc comparison all days).

Placebo monkeys did not display significant changes from baseline on any day except day 53. In conclusion, monkeys in the oral ropinirole and implanted ropinirole groups showed significant continuous improvement over the course of the study, while the placebo group did not (Figure 2).

\subsection{Global Dyskinesia Scale Scores}

One monkey in the oral ropinirole group showed a score of 1 on three of nine survey days. No monkeys in the ropinirole implant or placebo groups showed signs of dyskinesia. Given the small number of events, no statistical analyses were performed and no conclusions were drawn from the dyskinesia scale data.

\subsection{Skin Irritation}

No skin irritation was seen at the implant site in any monkey among the treatment groups during the study.

\subsection{Activity Monitoring Data}

Weekday Results (Wednesday-Friday): Animals receiving placebo showed a large and consistent reduction in overall activity compared with the baseline pre-MPTP levels. Animals receiving oral ropinirole showed alternating periods of significantly higher than normal activity directly following oral administration, interspersed with normal activity levels that correspond with the administration of the drug treatment (light phase). Oral treatment group had activity levels comparable to preMPTP levels overnight (dark phase). However, monkeys receiving the ropinirole implant demonstrated activity levels comparable to baseline during the day, with a slight increase in activity compared to baseline at nighttime. Figure 3 illustrates the average weekday activity by hour as measured by the AMS. In addition to the 3 treatment groups, there is a fourth line that represents the baseline AMS taken before the MPTP-Induction phase, and is therefore representative of the monkeys' normal, pre-Parkinsonian activity levels (Figure 3).

Weekend Results (Saturday-Sunday): When not receiving treatment over the weekend, monkeys in the oral ropinirole group displayed activity levels similar to that of placebo treatment in MPTP-treated monkeys. The activity levels of those in the ropinirole implant group, however, remained similar to that of their weekday period. Figure 4 illustrates the average activity of the monkeys within each group over the weekends. The fourth line represents weekend activity before the MPTP-Induction phase, and the average pre-Parkinsonian activity levels of the monkeys at night (Figure 4).

\section{Discussion}

The current methods of drug administration for Parkinson's Disease require multiple doses medication per day. These dosing regimens often result in cycles of brief symptom remission interspersed with periods of bradykinesia and dyskinesia, making normal function difficult as the medication wears off between doses. Ropinirole implants have the potential to eliminate these socalled "off" periods experienced by Parkinsonian patients between oral doses. Data in the current study indicate that functional recovery on the primary outcome CRS measure was similar inthe ropinirole implant and oral ropinirole groups duringtreatment days 11 through 60 . In addition, the oral group showed significant improvement in CRS as early as day 4. Although there were no assessments between days 4 and 11, we cannot rule out the possibility that implants require a lag of up to 11 days to become effective. Notably, the ropinirole implant was effective with doses 1/9th of the daily oral doses, suggesting that low-dose continuous dosing may provide comparable efficacy with lower potential for side effects.

The activity monitoring data indicate that treatment with ropinirole implants resulted in a consistent pattern of activity, yielding a pattern similar to that seen prior to MPTP treatment. This similarity was present both during the weekdays and on the weekends. Conversely, on weekdays, dosing of oral ropinirole yielded a high degree of variability, with higher values following oral dosing relative to pre-MPTP levels. As hyperactivity in animals has excellent predictive validity for psychosis and mania in humans, these data are consistent with the clinical observation that oral ropinirole can cause periods of frank psychosis (hallucinations and delusions), and mania-like behaviors including hyper-sexuality, gambling and aggression at minimum effective oral doses [10-12]. Ropinirole implants did not, however, yield hyperactivity at clinically (CRS scores) effective doses. This suggests that implants may be able to reduce the disabling iatrogenic psychiatric side effects of dopamine agonists. This was true despite the observation that both treatment groups had comparable Clinical Rating Scale scores during the active treatment period.

The oral ropinirole group showed activity levels similar to placebo during the weekends, consistent with off periods between doses in patients. There was also greater 


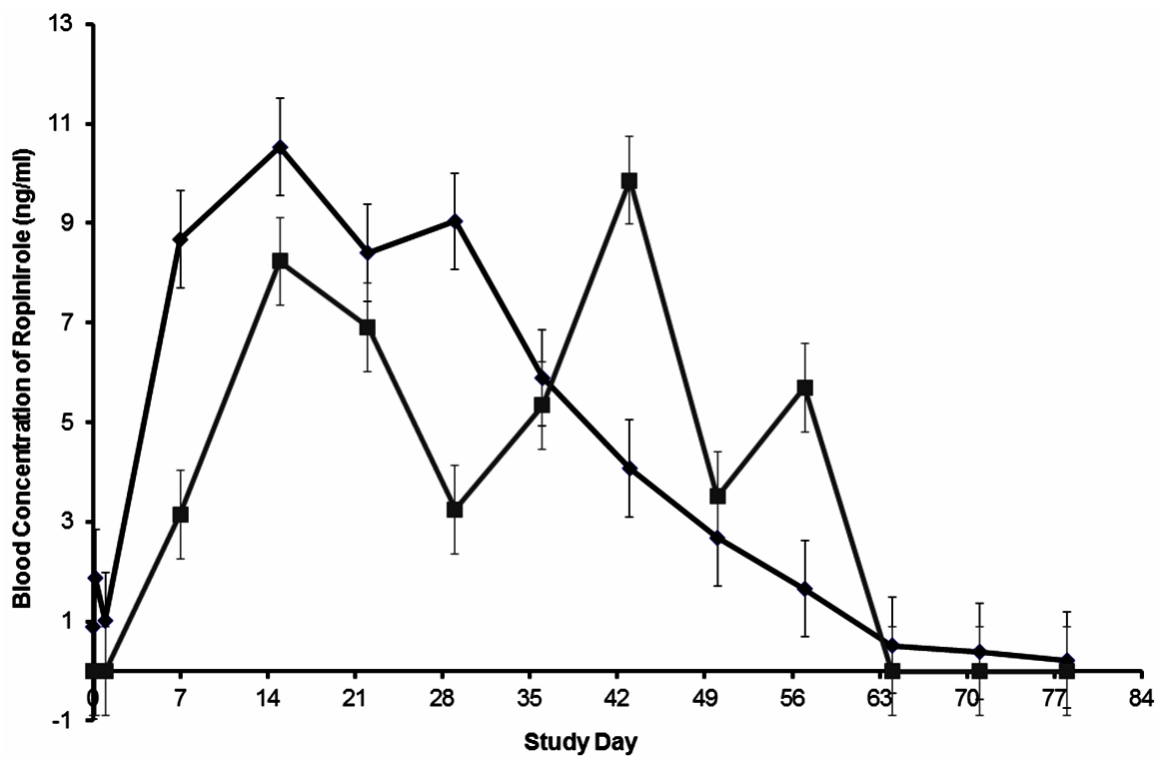

Figure 1. Serum ropinirole concentration. Serum ropinirole concentration is shown in each treatment group across all study days. Samples were taken once each week in the orally medicated group (gray) and ropinirole implant group (black). The data reveal a peak concentration of ropinirole in the implant group at 14 days post-implantation, as well as a slow and steady decline in concentration of the drug following day 28. The implant and the oral groups show comparable pharmacokinetic concentrations of ropinirole between days 7 and 49 .

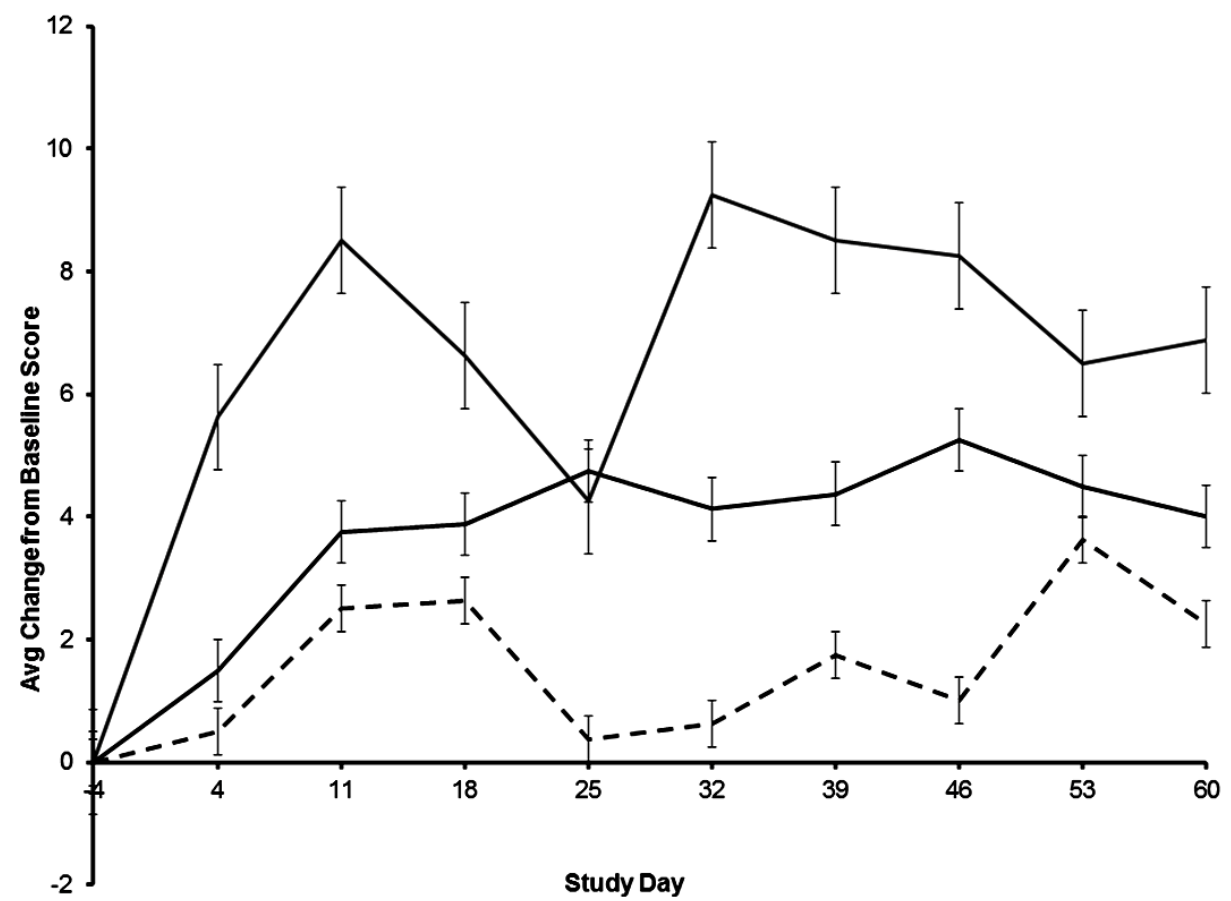

Figure 2. Clinical rating scale scores. Average change from baseline Clinical Rating Scale score are shown for each treatment group across time. The orally medicated group is shown in the solid gray line, the ropinirole implant group is in the solid black line and the control group is shown as the dotted black line. Monkeys in each group were assessed by the CRS once each week throughout the treatment period on days 4 - 60. The baseline score is featured as day 4 , i.e. 4 days before the treatment began. The data reveal a significant interaction of week and treatment group, indicating that the groups' scores changed differently over the course of the study (pooled MS $=19.325, F=1.796,(18,8), p=0.04)$. The ropinirole implant group showed continuous significant improvement from baseline scores on days 11 through 60 (p $<0.05$ Tukey's post-hoc comparison all days). Monkeys receiving oral ropinirole showed significant continuous improvement from baseline scores on all days during which drug was administered ( $p<0.05$ Tukey's post-hoc comparison all days). Placebo monkeys did not display significant changes from baseline on any day except day 53. 

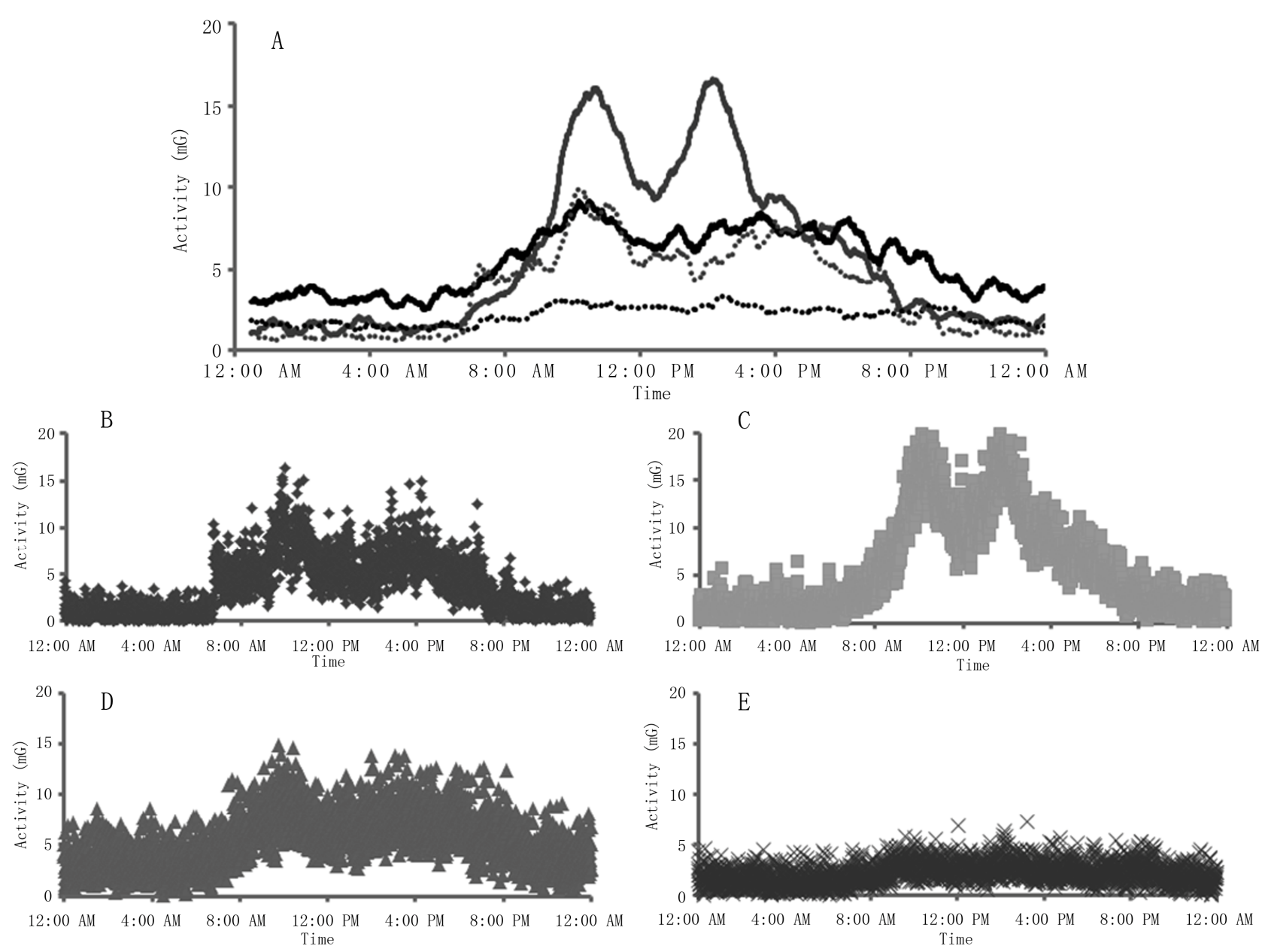

Figure 3. 24 hour weekday activity. (A) Average 24 hour activity by treatment group is shown from Wednesday through Friday. Oral medication was only given during the weekdays and activity is not shown on Monday and Tuesday as these days were used for PK sampling and behavioral assessments. Oral treatments (medication or placebo) were given at 8 am, 12 pm, and $4 \mathrm{pm}$. The average activity of the orally medicated group (solid gray), ropinirole implant (solid black) and control group (dashed black) are shown in comparison to the average activity of all monkeys before being rendered bilaterally Parkinsonian with MPTP (dashed gray). Control animals showed a large and consistent reduction in overall activity compared with the baseline pre-MPTP levels. The oral ropinirole group showed alternating periods of higher than normal activity directly following oral administration, interspersed with normal activity levels during the day, and had activity levels comparable to pre-MPTP levels overnight. The ropinirole implant group demonstrated activity levels comparable to pre-MPTP baseline during the day, with a slight elevation of activity compared to baseline at night; (B) All points of Pre-MPTP induction activity for the $\mathbf{2 4}$ hour day from Wednesday through Friday; (C) All points of 24 hour activity for the orally medicated group from Wednesday through Friday; (D) All points of 24 hour activity for the ropinirole implant group from Wednesday through Friday; (E) All points of 24 hour activity for the control group from Wednesday through Friday.

variability in the oral group relative to implants for CRS, activity and ropinirole plasma levels during the treatment phase. These data suggest that implants may lead to more consistent efficacy in humans than is possible with traditional oral regimens.

Monkeys in the ropinirole implant group exhibited elevated levels of activity at night compared to their own pre-MPTP induction levels and compared to monkeys in both oral and placebo groups. Whether the monkeys were waking throughout the night or turning in their sleep is not known as there was no observation at night. However, the monkeys in this group did not display unusual behavior during the day, and notably, their weight did not fall out of a healthy range during the course of the study. This suggests that they were getting an adequate amount of sleep during treatment.

Although dyskinesias were too rare to draw conclusions in the current study, dyskinesia has been linked to fluctuations in dopamine activity in the brain, and is important to understanding the potential of the sustained delivery implant. Future experiments might determine how the consistent drug delivery of the ropinirole implant compares to the intermittent delivery of oral medication in eliminating dyskinesias. 

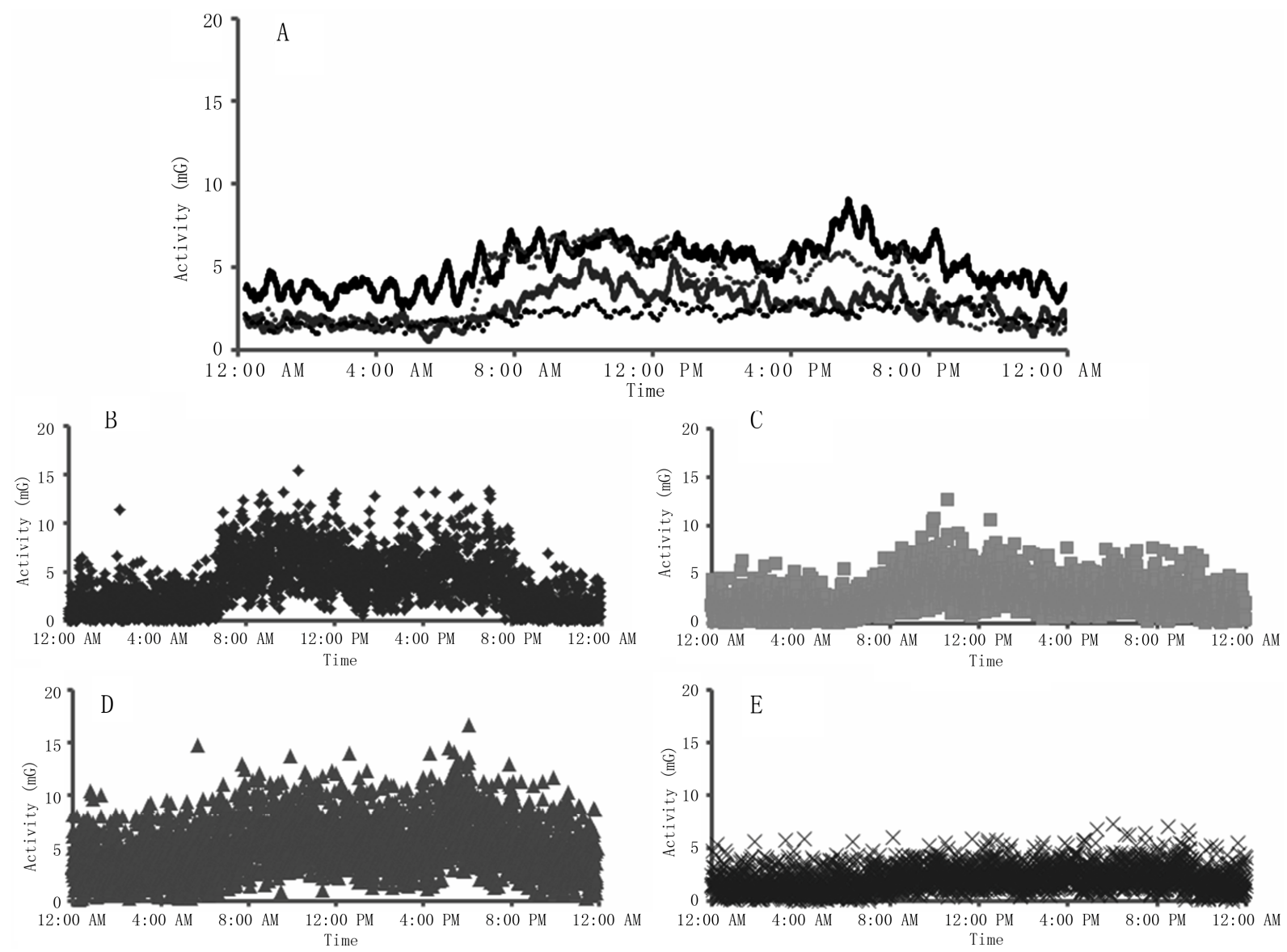

Figure 4. 24 hour weekend activity. (A) Average 24 hour activity by treatment group on Saturday and Sunday. Oral medication was not given on the weekend to assess potential carry over effects of weekday treatment. Average activity of the orally medicated group (solid gray), ropinirole implant group (solid black), control group (black dashed) are shown in relation to the pre-MPTP baseline (gray dashed). Monkeys in the oral ropinirole group displayed activity levels similar to that of placebo treatment over the weekend. However, the activity level of those in the ropinirole implant group remained similar to that of their weekday period and was similar to pre-MPTP baseline levels; (B) All points of average Pre-MPTP 24 hour activity from Saturday and Sunday, measured by each 30 second epoch; (C) All points of average 24 hour activity for the orally medicated group on Saturday and Sunday; (D) All points of average 24 hour activity for the ropinirole implant group on Saturday and Sunday; (E) All points of 24 hour average activity for the control group on Saturday and Sunday.

\section{Conclusion}

In summary, the ropinirole implant yielded comparable efficacy, as measured by CRS scores, to oral ropinirole medication. Activity levels in the monkeys with a ropinirole implant more closely resemble the baseline "normal" condition, than those resulting from oral medication. These data also suggest that low dose ropinirole implants have the potential to provide clinical improvement in bradykinesia with less "off" periods and lower risk for medication induced psychosis and other psychiatric symptoms. Future studies can address the extent to which ropinirole implants may reduce risk of medication-induced dyskinesia.

\section{Acknowledgements}

This study was supported by NuPathe (JHK, LL, CO, TS,
MP). JHK also supported in part by the Parkinson's Disease Foundation. SJS, LN and RL supported by R01MH074672. SJS, TS and JHK have served as consultants to NuPathe and MP and CO are employees of NuPathe. SJS has also served as a consultant to or grant recipient from Lundbeck, Astra Zeneca, Abbott, and Astellas. JHK is a founding scientist with a financial interest in Ceregene Inc., USA.

\section{REFERENCES}

[1] E. M. L. Kelly, L. Collins and P. G. Patil, "Deep Brain Stimulation for Movement Disorders," Neurobiology of Disease, Vol. 38, No. 3, 2010, pp. 338-345.

[2] I. Ferrer, "Neuropathology and Neurochemistry of Nonmotor Symptoms in Parkinson's Disease,” Parkinsons Disease, 2011, Article ID: 708404. 
[3] C. H. Adler, K. D. Sethi, R. A. Hauser, et al. and The Ropinirole Study Group, "Ropinirole for the Treatment of Early Parkinson's Disease,” Neurology, Vol. 49, No. 2, 1997, pp. 393-399. doi:10.1212/WNL.49.2.393

[4] S. Badarny, A. Stern, Z. Susel, et al., "Effect of Ropinirole on Visuo-Motor Test in Newly Diagnosed Parkinson's Disease Patients," Acta Neurologica Scandinavica, Vol. 113, No. 5, 2006, pp. 294-300.

[5] B. R. Boothman and E. G. Spokes, "Pharmacokinetic Data for Ropinirole,” Lancet, Vol. 336, No. 8718, 1990, p. 814.

[6] D. Knobel, S. Aybek, C. Pollo, et al., "Rapid Resolution of Dopamine Dysregulation Syndrome (Dds) after Subthalamic Dbs for Parkinson Disease (Pd): A Case Report," Cognitive Behavioral Neurology, Vol. 21, No. 3, 2008, pp. 187-189. doi:10.1097/WNN.0b013e318185e6e2

[7] J. M. Bostwick, K. A. Hecksel, S. R. Stevens, et al., "Frequency of New-Onset Pathologic Compulsive Gambling or Hypersexuality after Drug Treatment of Idiopathic Parkinson Disease,” Mayo Clinic Proceedings, Vol. 84, No. 4, 2009, pp. 310-316.
[8] M. L. Hans, L. Dan, K. I. Winey, et al., "Daily to Annual Biodegradable Drug Delivery Strategies for Psychoactive Compounds,” In: S. K. Mallapragada, Ed., Handbook of Biodegradable Polymeric Materials \& Applications, American Scientific Publishers, Stevenson Ranch, 2004, pp. 77-102.

[9] C. Rabin, Y. Liang, R. S. Ehrlichman, et al., "In Vitro and in Vivo Demonstration of Risperidone Implants in Mice," Schizophrenia Research, Vol. 98, No. 1-3, 2008, pp. 6678. doi:10.1016/j.schres.2007.08.003

[10] L. C. Amann, M. J. Gandal, T. B. Halene, et al., "Mouse Behavioral Endophenotypes for Schizophrenia,” Brain Research Bulletin, Vol. 83, No. 3-4, 2010, pp. 147-161.

[11] J. M. Bostwick, K. A. Hecksel, S. R. Stevens, et al., "Frequency of New-Onset Pathologic Compulsive Gambling or Hypersexuality after Drug Treatment of Idiopathic Parkinson Disease,” Mayo Clinic Proceedings, Vol. 84, No. 4, 2009, pp. 310-316.

[12] A. E. Schrag, D. J. Brooks, E. Brunt et al., "The Safety of Ropinirole, a Selective Nonergoline Dopamine agonist, in Patients with Parkinson's Disease," Clinical Neuropharmacology, Vol. 21, No. 3, 1998, pp. 169-175. 